\title{
Pressure-induced structural change of intermediate-range order in poly(4-methyl-1-pentene) melt
}

\section{$\operatorname{AUTHOR}(S)$ :}

Chiba, Ayano; Funamori, Nobumasa; Nakayama, Kazuya; Ohishi, Yasuo; Bennington, Stephen; Rastogi, Sanjay; Shukla, Anuj; Tsuji, Kazuhiko; Takenaka, Mikihito

\section{CITATION:}

Chiba, Ayano ... [et al]. Pressure-induced structural change of intermediate-range order in poly(4-methyl-1-pentene) melt. Physical Review E 2012, 85(2): 021807.

\author{
ISSUE DATE: \\ 2012-02 \\ URL: \\ http://hdl.handle.net/2433/153288 \\ RIGHT: \\ (C)2012 American Physical Society
}




\title{
Pressure-induced structural change of intermediate-range order in poly(4-methyl-1-pentene) melt
}

\author{
Ayano Chiba, ${ }^{1, *}$ Nobumasa Funamori, ${ }^{2, \dagger}$ Kazuya Nakayama, ${ }^{1,2}$ Yasuo Ohishi, ${ }^{3}$ Stephen M. Bennington, ${ }^{4}$ \\ Sanjay Rastogi, ${ }^{5}$ Anuj Shukla, ${ }^{6}$ Kazuhiko Tsuji, ${ }^{1}$ and Mikihito Takenaka ${ }^{7, \sharp}$ \\ ${ }^{1}$ Department of Physics, Keio University, Yokohama 223-8522, Japan \\ ${ }^{2}$ Department of Earth and Planetary Science, University of Tokyo, Tokyo 113-0033, Japan \\ ${ }^{3}$ Japan Synchrotron Radiation Research Institute (SPring-8), Hyogo 679-5198, Japan \\ ${ }^{4}$ Rutherford Appleton Laboratory, Chilton, Didcot OX11 OQX, United Kingdom \\ ${ }^{5}$ Department of Materials, Loughborough University, Loughborough, LE11 3TU, United Kingdom \\ ${ }^{6}$ European Synchrotron Radiation Facility, 6 rue Jules Horowitz, F-38043 Grenoble Cedex 9, France \\ ${ }^{7}$ Department of Polymer Chemistry, Kyoto University, Kyoto 615-8510, Japan
}

(Received 7 September 2011; revised manuscript received 1 January 2012; published 27 February 2012)

\begin{abstract}
High-pressure in situ x-ray diffraction and specific-volume measurements on isotactic poly(4-methyl-1pentene) melt have uncovered abrupt changes in the pressure dependence of microscopic structure as well as that of macroscopic density. The first sharp diffraction peak of the polymer melt, which is related to the intermediate-range order and is explained as resulting from the correlations between main chains, is suppressed at pressures less than $1 \mathrm{kbar}$. These changes in intermediate-range order show similarities to those seen in liquid-liquid or amorphous-amorphous transitions in simpler small molecule based systems, suggesting that this kind of phenomenon may occur in a wide range of materials.
\end{abstract}

DOI: 10.1103/PhysRevE.85.021807

PACS number(s): 61.25.hk, 62.50.-p, 64.70.Ja

\section{INTRODUCTION}

There has been great excitement in recent years about the discoveries of liquid-liquid transitions (LLTs) in systems with small molecular or structural units, such as those seen in water [1,2], sodium [3,4], silicon [5], phosphorus [6], arsenic [7], and many others [7-10]. These transitions (which include continuous transformations) are characterized by changes in static structure. In contrast, the understanding of LLTs in polymers remains insufficient and confusing. This is due in part to the fact that most of the studies have focused on a certain type of transition, which intrinsically relates to changes not in static structure but in relaxation phenomena. These changes occur in a variety of glass forming polymers, and presumably in any other glass forming systems, at relatively low temperatures around 1.2 times glass transition temperature $T_{g}$ (hereafter $1.2 T_{g}$ transition) $[11,12]$. Some studies have reported static changes which are not associated with $1.2 T_{g}$ transitions $[13,14]$. In these studies, however, measurements have been conducted only as a function of temperature. To investigate changes in static structure, measurements as a function of pressure are preferable because applying pressure can alter interatomic potentials more directly. Such measurements have been proven to be successful in simper systems [2,3,5-9], and therefore are indispensable for a better understanding of LLTs in polymers.

Amorphous-amorphous transitions (AATs) have also been discovered in materials such as ice [15] and $\mathrm{SiO}_{2}$ glass [16, 17]. Almost all the materials showing LLTs or AATs have anomalous melting curves and there is a widely held belief that if the melting curve shows a maximum as a function of pressure, then this must be related to a structural change in

\footnotetext{
*ayano@phys.keio.ac.jp

${ }^{\dagger}$ funamori@eps.s.u-tokyo.ac.jp

†takenaka@alloy.polym.kyoto-u.ac.jp
}

liquid [18]. For example, cesium and tellurium show LLTs near the melting curve maximum that is thought to be caused by pressure-induced changes in bonding that result in changes in coordination number $[9,18]$. However, LLTs or AATs can also be caused by changes in bond angle or packing as is the case in $\mathrm{SiO}_{2}$ glass around $100 \mathrm{kbar}[16,17]$. This kind of changes seen in the intermediate-range order, which is a structural order intermediate between short-range discrete chemical bonds and long-range crystalline lattices, are likely to be important in polymers.

In this study, we chose to look at isotactic poly(4-methyl-1pentene) (P4MP1), which has a maximum in its melting curve at $1.5 \mathrm{kbar}$ (Fig. 1) [19,20], to explore LLTs in polymers at high enough temperatures, compared to its melting temperature of $240{ }^{\circ} \mathrm{C}$ (and $1.2 T_{g} \approx 90^{\circ} \mathrm{C}$ ) [19-21]. P4MP1 has a remarkably low density at ambient pressure [22,23]. Its crystalline phase $\mathrm{I}$ in Fig. 1 has a large interchain C-C distance [23-25] and an unusually high permeability for gases such as $\mathrm{CO}_{2}$ and $\mathrm{CH}_{4}$ [24] implying a structure with significant void space, due to the loosely packed bulky side chains. The melt has an even lower density [22], suggesting that there are even larger amounts of space around the side chains in the melted state. By increasing pressure, this void space will be squeezed and a structural change in intermediate-range order may well be triggered.

\section{EXPERIMENT}

Isotactic P4MP1 (Scientific Polymer Product Inc.) was kept at $280{ }^{\circ} \mathrm{C}$ and $3 \times 10^{-6}$ Torr for $3 \mathrm{~h}$ for degasification [21] and then molded into pellets having dimensions of 2.0 $\mathrm{mm}$ in diameter and $1.0 \mathrm{~mm}$ in thickness at $255{ }^{\circ} \mathrm{C}$ with pure-aluminum mold plates. The impurity due to this molding was confirmed to be low, $<50 \pm 30 \mathrm{ppm}$ by weight, by atomic absorption spectrometry [26,27]. The pellets thus obtained were transparent and one of them was used as a sample in X-ray diffraction measurements. Unmolded P4MP1 having larger 


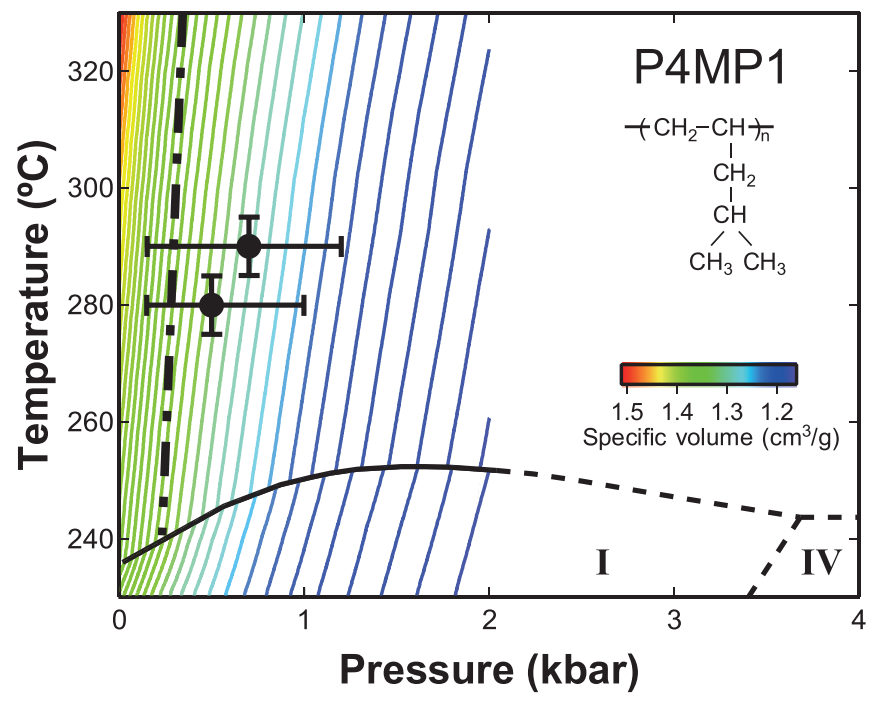

FIG. 1. (Color) Phase diagram of P4MP1. The results of specificvolume measurements are shown by the colored contours. The break in these measurements that indicates melting is drawn as a solid black line. The low- and high-density crystalline phases are indicated with I and IV, respectively [19]. The black circles show the abrupt changes seen in the pressure dependence of $x$-ray diffraction profiles [Figs. 4(a) and 4(b)] and the dashed-dotted line shows those seen in specific-volume data [Fig. 4(c)].

dimensions, $9.0 \mathrm{~mm}$ in diameter and $15 \mathrm{~mm}$ in thickness, was used as a sample in specific-volume measurements.

High-pressure in situ $\mathrm{x}$-ray diffraction measurements were conducted at two temperatures, 280 and $290{ }^{\circ} \mathrm{C}$, at the beamline BL10XU of SPring-8, Japan [28]. A new diamond piston-cylinder cell (Fig. 2) was designed to measure twodimensional diffraction profiles under high pressure and temperature. This cell is optimized to measure polymers consisting of light elements whose atomic scattering factors are small; i.e., it enables a compression without too much squeeze (a)

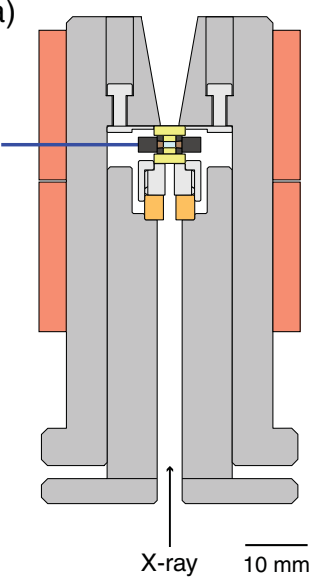

(b)

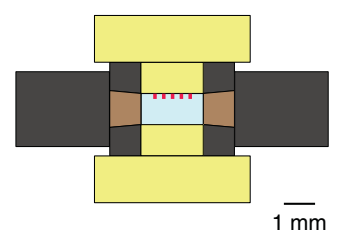

FIG. 2. (Color) Schematic illustrations of the cross section of the diamond piston-cylinder high-pressure cell. (a) Whole picture of the cell. (b) Enlarged picture of the central part of the cell. Inner piston and cylinder were made of synthetic $\mathrm{Ib}$ single-crystal diamond and heat resistant tungsten carbide alloy. $\mathrm{SrB}_{4} \mathrm{O}_{7}: \mathrm{Sm}^{2+}$ pressure maker and $\mathrm{K}$-type (chromel-alumel) thermocouple were used. of the sample thickness, which would otherwise crucially reduce the intensity of diffraction data. The cell was set in a lever-arm body and compressed to generate high pressures [29]. An external-resistance heating technique was used to generate high temperatures [29]. X-ray diffraction profiles were recorded on an imaging-plate detector. The energy and diameter of x-ray beam were $40 \mathrm{keV}$ and $0.1 \mathrm{~mm}$, respectively. The camera length was $450 \mathrm{~mm}$, and exposure time was $1 \mathrm{~min}$ for each profile.

In the diffraction experiments, pressure was determined by measuring the shift of the ${ }^{7} D_{0^{-}}{ }^{5} F_{0}$ fluorescence line of $\mathrm{SrB}_{4} \mathrm{O}_{7}: \mathrm{Sm}^{2+}$ [30]. This fluorescence line has advantages in determining pressure at high temperatures, because it is a single narrow line and it shows only a slight broadening and shift with temperature. Small pieces of $\mathrm{SrB}_{4} \mathrm{O}_{7}: \mathrm{Sm}^{2+}$ were set between the sample and the diamond piston. The fluorescence was measured by a spectrometer having a focal length of $250 \mathrm{~mm}$, with an 1800-grooves/mm grating. A charge-coupled device of 1024 pixels $\times 128$ pixels format, with each pixel having an area of $27 \mu \mathrm{m} \times 27 \mu \mathrm{m}$, was used as a detector. A pixel size of $27 \mu \mathrm{m}$ corresponds to a wavelength of $0.0247 \mathrm{~nm}$. The shift of the fluorescence line was obtained by fitting Gaussian to each peak and then pressure was calculated by using a conversion factor $\Delta \lambda / \Delta P=0.0255 \mathrm{~nm} / \mathrm{kbar}$ [30]. The standard deviation of fitting was typically $0.003 \mathrm{~nm}$, which corresponds to $0.1 \mathrm{kbar}$; i.e., the precision of pressure is estimated to be $\pm 0.1 \mathrm{kbar}$. On the other hand, the accuracy of pressure may be as low as $\pm 0.4 \mathrm{kbar}$. It is limited mainly due to the following two reasons: (1) It was difficult to know the exact peak position at $280-290{ }^{\circ} \mathrm{C}$ and ambient pressure in the same experimental setup in which high-pressure experiments were conducted, because setting the sample in the cell and raising temperature inevitably caused a pressure increase. (2) The conversion factor at room temperature was used in substitution for that at high temperatures (because the temperature dependence of this factor is not known very well). The difference in the peak positions between the two literatures for the $\mathrm{SrB}_{4} \mathrm{O}_{7}: \mathrm{Sm}^{2+}$ scale [30] is $\pm 0.01 \mathrm{~nm}$ at $280-290{ }^{\circ} \mathrm{C}$ and ambient pressure, and this corresponds to $\pm 0.4 \mathrm{kbar}$, which is comparable to the estimated accuracy of pressure.

Specific-volume measurements were conducted with a metal-bellows technique [31]. This is a well-established technique and the accuracy of the measurements is very high; $0.2 \%$ for pressure, $\pm 1{ }^{\circ} \mathrm{C}$ for temperature, and $\pm 0.0015 \mathrm{~cm}^{3} / \mathrm{g}$ for specific volume [31]. The pressure dependence of the volume was measured at temperatures between 230 and $340{ }^{\circ} \mathrm{C}$. The melting curve of P4MP1 was so far measured by a few groups $[19,20]$, and all of them show a maximum as a function of pressure. Our results based on the volume measurements also show a maximum (Fig. 1). The melting point of our sample at ambient pressure was also measured by differential scanning calorimetry. The result by this method shows good agreement with that based on the volume measurements.

\section{RESULTS AND DISCUSSION}

We performed in situ $\mathrm{x}$-ray diffraction measurements with increasing and decreasing pressure at two temperatures, 280 and $290^{\circ} \mathrm{C}$. An example of two-dimensional raw data is shown in Fig. 3. The arrow (i) shows a peak around $0.6 \AA^{-1}$, which 


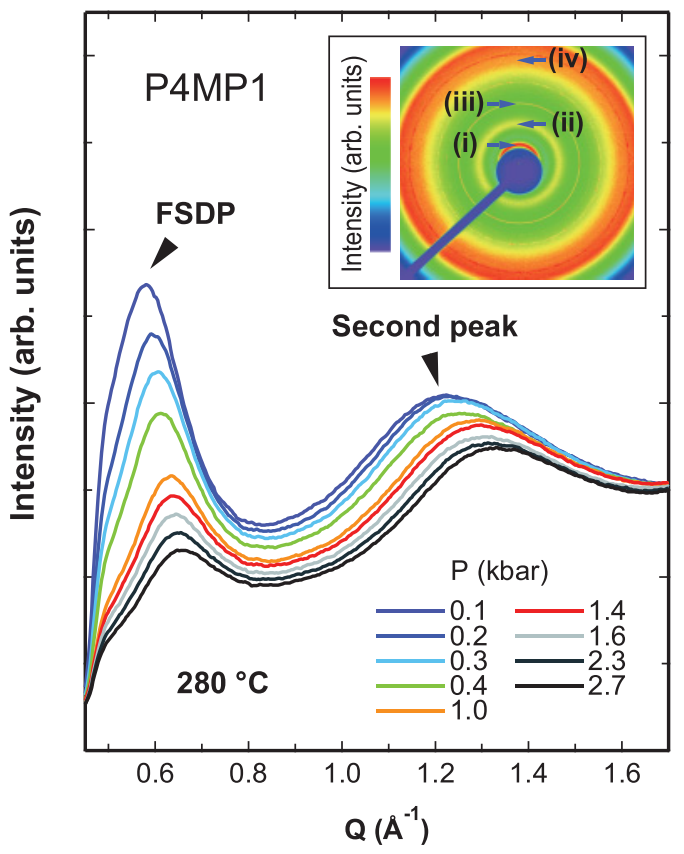

FIG. 3. (Color) X-ray diffraction patterns of P4MP1 melt. An example of the two-dimensional diffraction pattern (raw data at $0.1 \mathrm{kbar}$ ) is shown in the inset. The peak (i) shows the FSDP, (ii) the second peak, (iii) a diffraction line from a graphite gasket, and (iv) Compton scattering from diamond pistons.

we call the first sharp diffraction peak (FSDP), and the arrow (ii) shows the second peak around $1.2 \AA^{-1}$. The intensity of the FSDP decreases with pressure (Fig. 3) at both the temperatures measured, as does the ratio of the intensities of the FSDP to the second peak [Fig. 4(a)] [32]. But more significantly, there are abrupt changes in the slope in this ratio at 0.5 and $0.7 \mathrm{kbar}$ at 280 and $290^{\circ} \mathrm{C}$, respectively, and in the position of the FSDP as a function of pressure [Fig. 4(b)]. All these observations are completely reversible [33]. Such a large clear change has not been observed in any other one-component polymer melts to date.

We also measured the specific volume by using a metalbellows technique [31] and this is shown in the contour plot in Fig. 1. In order to see the contraction visually, we have used the so-called $f-F$ plot [34], which is a normalized stress $(F)$-finite strain $(f)$ plot derived from the measured isothermal volume contraction; a plot that is commonly used in high-pressure science. Interestingly, as with the diffraction measurements, abrupt changes are seen in the slope [Fig. 4(c)], indicating anomalous increases in the bulk modulus. According to the well-known definition of the Ehrenfest classification, the break in the second derivatives of the thermodynamic free energy (i.e., bulk modulus in this case) indicates the second-order transition. However, it may be more probable that these changes are due to the continuous but sharp transformation at high temperatures above the liquid-liquid critical point $[18,35]$. In any case, based on the $f-F$ plot, the apparent boundary between low-density and high-density melts can be drawn as shown by the dashed-dotted line in Fig. 1. It coincides, within the margin of error, with the same boundary seen in the diffraction results shown by black circles [36]. It is important to note that the boundary between two melts is almost perpendicular to the pressure axis, suggesting that the density is the key control parameter for the structural transition.

Among all results above, the most notable is the change of FSDP. In systems with small molecular or structural units, changes in FSDP often relate to LLTs or AATs. In fact, the changes that we see in P4MP1 show intriguing similarities to those seen in $\mathrm{SiO}_{2}$ glass [17]. In $\mathrm{SiO}_{2}$ glass, the intensity of FSDP is high when the density is low. As the structure densifies, its intensity decreases and its position shifts to higher momentum transfers. Above the AAT, the rate of change of the FSDP is significantly lower. Although the origin of the FSDP may not be identical, these similarities suggest the universal
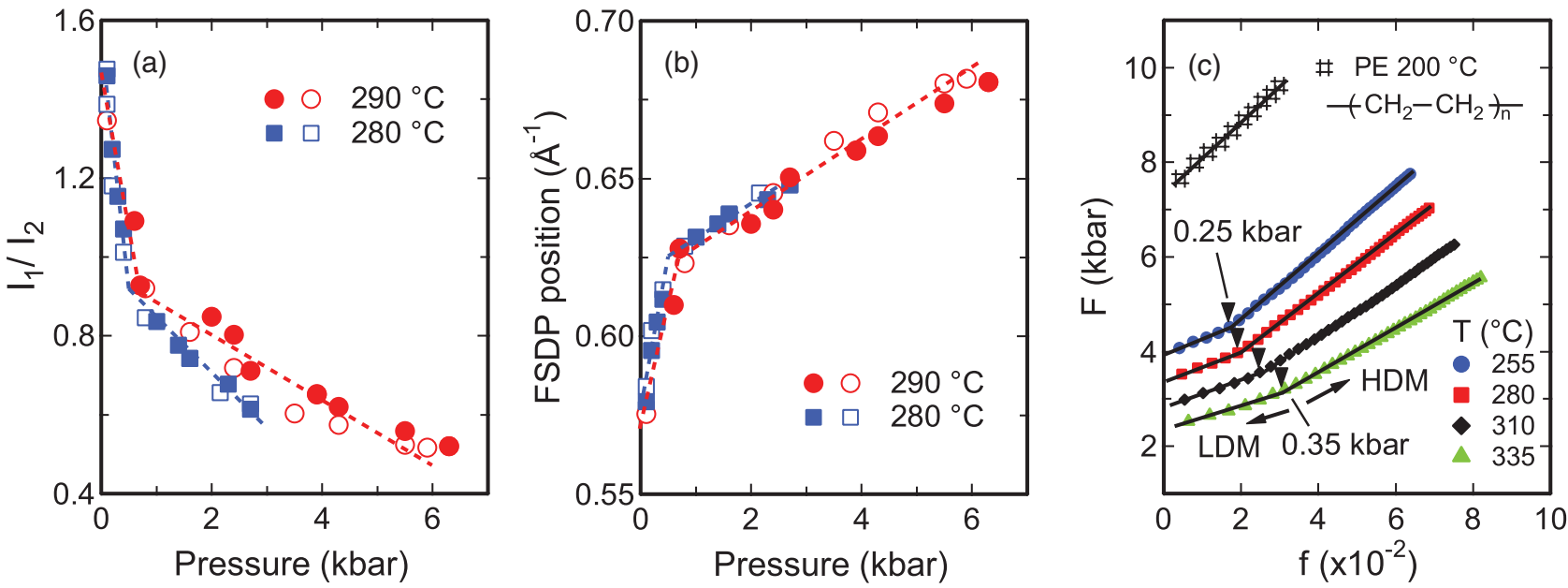

FIG. 4. (Color) Pressure dependence of FSDP and specific volume of P4MP1 melt. (a) The ratio of the intensities of the FSDP ( $\left.I_{1}\right)$ to that of the second peak $\left(I_{2}\right)$ and (b) the position of the FSDP. Filled symbols show the data taken under increasing pressure and open symbols under decreasing pressure. (c) The normalized stress $(F)$-finite strain $(f)$ plot for P4MP1 melt extracted from the specific-volume measurements. The arrows show abrupt changes in the slopes, corresponding to the boundary between the low-density melt (LDM) and the high-density melt (HDM). The same plot for polyethylene melt [47] is shown for comparison. Lines are guides for the eyes. 
(a) LDM

(b) HDM

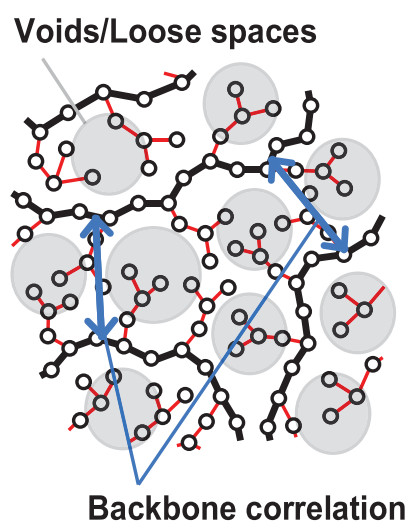

FIG. 5. (Color) Schematic pictures of possible structures of P4MP1 melt. (a) The low-density melt, LDM, and (b) the high-density melt, HDM. Small black circles indicate carbon atoms, and black thick and red thin lines indicate main and side chains, respectively. Blue arrows indicate correlations between backbones (main chains) and gray large circles indicate void spaces, both of which would cause the intermediate range order.

behavior that is related to the change in the packing of the network-forming units.

Here, we explore the links between the fields of liquid and amorphous science and polymer science by looking at the similarities in the behavior and interpretation of the FSDP in both fields. In the liquid and amorphous communities, the peak is called FSDP, and is known to be related to the intermediaterange order [37-40]. The equivalent peak in polymeric systems is known as the polymerization peak $[41,42]$ or larger than van der Waals peak [43] (or low van der Waals peak [44]). The polymerization peak does not appear in every polymer melt (for example, it is absent in polyethylene melt [14,25,45]), and it is not seen in monomeric systems $[41,42]$. Whereas the higher momentum-transfer peak is observed in all polymer glasses and melts and as such it is normally called the main peak $[25,42]$ or van der Waals peak $[43,44]$. Similarly, the FSDP is not always present in simper systems. But it is present in many, including $\mathrm{SiO}_{2}$ glass [17,40], liquid phosphorus [6], and others [37-39]. Also, as in polymeric systems, the higher momentum-transfer peak is found in all glasses and melts.

The usual interpretation for the FSDP of simpler systems is that it is due to the periodicity of boundaries between a succession of cages $[39,40]$ or the chemical ordering of interstitial voids around the clusters of atoms [38]. In polymers, the polymerization peak is usually assigned to backbonebackbone correlation [25,41-46], which could also be thought of as a correlation of dense backbones surrounding voids that are sparsely filled by the side chains; a picture that is particularly true for P4MP1, because the side chains are bulky and inefficiently packed [23-25]. In fact, the polymerization peak in our measurements occurs at $0.6 \AA^{-1}$, which corresponds to $2 \pi /\left(0.6 \AA^{-1}\right) \approx 10.5 \AA$ in real space, which is consistent with the expected interbackbone distance for the melt (the distance is evaluated from that for the crystalline state [23-25]). Thus the origin of this polymerization peak resembles that of the FSDP of simpler systems; voids correspond to the loose space for the side chains, and frameworks of cages correspond to the backbone. For these reasons, we regard the polymerization peak and the FSDP to manifestations of the same type of noncrystalline structure and we refer to it as the FSDP.

Given that the origin of the FSDP is related to the correlation between backbones [Fig. 5(a)] [25,41-46], we can assume that the rapid decrease in the intensity of the FSDP with pressure [Fig. 4(a)] indicates a disordering of backbone-backbone correlations and the shift to higher momentum transfers [Fig. 4(b)] indicates a reduction of the void spaces between backbones. In other words, the high compressibility we see in the low-density melt is due to compaction of the void space which suppresses the backbone-backbone correlations [Figs. 5(a) and 5(b)]. It is interesting to compare the data of P4MP1 melt to that of polyethylene melt [47], which does not show any significant changes in the slope [Fig. 4(c)]. Polyethylene does not have any side chains to create a void structure so its density is higher and it is significantly less compressible at low pressures, but has a similar compressibility at high pressures.

Anomalous behavior of the FSDP of polystyrene has been reported in measurements as a function of temperature $[12,46]$, although the reversibility may not have been confirmed. The temperatures at which the anomaly has been observed range from 90 to $160{ }^{\circ} \mathrm{C}$ and show a strong correlation with $T_{g}$ of the sample $\left(T_{g} \approx 40-90{ }^{\circ} \mathrm{C}\right)$ [12]. Therefore this anomaly may be associated with $1.2 T_{g}$ transition [11,12], which is induced by temperature. In contrast, the change seen in P4MP1 is induced by pressure (Fig. 1). Moreover, it occurs at high temperatures of about 1.8 times $T_{g}$. Therefore these anomalies in polystyrene and P4MP1 appear to be of an intrinsically different character. At the same time, a change similar to that in P4MP1 may also occur in polymers having an FSDP (including polystyrene). This is especially the case for syndiotactic polystyrene because it also has a maximum in its melting curve [48]. Furthermore, anomalies in the temperature dependence of static structures have been reported in some polymer melts (including polyethylene, polystyrene, and P4MP1 melts) at high enough temperatures $[13,14]$. Although the reported anomalies are less directly related to static structures or smaller than those we found in P4MP1 melts, they might suggest the occurrence of a wide variety of LLTs in polymers.

In this study, we found the evidence of the LLT (or continuous but sharp transformation) in P4MP1, which is characterized by a change in intermediate-range order. The fact that such a large change occurs in P4MP1 at easily accessible pressures is noteworthy, and originates from the nature of the loosely packed structure of the polymer. The connectivity of its monomeric units, its bulky side chains and its tacticity hinder the formation of a close packed structure, creating a material with large amounts of void space. This means that, unlike simpler materials which require relatively large energy changes in bond angle to remove void space, only small energy conformational changes are required. Such transitions may be relatively common even in more familiar polymers such as polystyrene, as discussed above. This and the modest pressures and temperatures at which these phenomena occur open up the intriguing possibility of using LLTs or AATs as an industrial process to control the physical properties of plastics. 


\section{ACKNOWLEDGMENTS}

The authors thank T. Matsukawa, T. Kawamoto, D. Wakabayashi, and scientists at beamlines BL10XU and ID02 for collaboration, and Y. Katayama and O. Yamamuro for advice. This work was supported by JSPS and MEXT, Japan, and KLL, Keio University. Preliminary experiments were performed at ID02/ESRF, France. Experiment numbers are 2010A1382 (SPring-8) and SC-2279 (ESRF).
[1] O. Mishima and H. E. Stanley, Nature (London) 396, 329 (1998); P. H. Poole et al., ibid. 360, 324 (1992).

[2] J. H. Eggert, G. Weck, and P. Loubeyre, J. Phys.: Condens. Matter 14, 11385 (2002); A. F. Goncharov et al., J. Chem. Phys. 130, 124514 (2009); Y. Katayama et al., Phys. Rev. B 81, 014109 (2010).

[3] E. Gregoryanz et al., Phys. Rev. Lett. 94, 185502 (2005).

[4] J.-Y. Raty, E. Schwegler, and S. A. Bonev, Nature (London) 449, 448 (2007).

[5] N. Funamori and K. Tsuji, Phys. Rev. Lett. 88, 255508 (2002).

[6] Y. Katayama et al., Nature (London) 403, 170 (2000).

[7] A. Chiba et al., Phys. Rev. B 80, 060201 (2009).

[8] K. Tsuji et al., Rev. Sci. Instrum. 60, 2425 (1989); V. V. Brazhkin and A. G. Lyapin, J. Phys.: Condens. Matter 15, 6059 (2003); Y. Katayama and K. Tsuji, ibid. 15, 6085 (2003); M. C. Wilding, M. Wilson, and P. F. McMillan, Chem. Soc. Rev. 35, 964 (2006); T. Hattori et al., Phys. Rev. B 76, 144206 (2007).

[9] K. Tsuji et al., J. Non-Cryst. Solids 117/118, 72 (1990); N. Funamori and K. Tsuji, Phys. Rev. B 65, 014105 (2001); S. Falconi et al., Phys. Rev. Lett. 94, 125507 (2005).

[10] A. Chiba, Y. Ohmasa, and M. Yao, J. Chem. Phys. 119, 9047 (2003); H. Tanaka, R. Kurita, and H. Mataki, Phys. Rev. Lett. 92, 025701 (2004); C. Otjacques et al., Phys. Rev. B 82, 054202 (2010).

[11] R. F. Boyer, J. Polym. Sci. Pol. Sym. 14, 267 (1966); W. Götze and L. Sjögren, Rep. Prog. Phys. 55, 241 (1992); S. S. N. Murthy, J. Polym. Sci. Pol. Phys. 31, 475 (1993); O. Yamamuro et al., J. Phys.: Condens. Matter 6, L169 (1994); V. N. Novikov and A. P. Sokolov, Phys. Rev. E 67, 031507 (2003).

[12] T. Hatakeyama, J. Macromol. Sci. Phys. B 21, 299 (1982).

[13] J. K. Krüger et al., Polymer 21, 620 (1980).

[14] V. Dobrescu and C. Radovici, J. Macromol. Sci. Chem. A 22, 983 (1985).

[15] O. Mishima, L. D. Calvert, and E. Whalley, Nature (London) 314, 76 (1985)

[16] M. Grimsditch, Phys. Rev. Lett. 52, 2379 (1984); R. J. Hemley et al., ibid. 57, 747 (1986); F. S. El'kin et al., JETP Lett. 75, 342 (2002).

[17] C. Meade, R. J. Hemley, and H. K. Mao, Phys. Rev. Lett. 69, 1387 (1992); Y. Inamura et al., ibid. 93, 015501 (2004); T. Sato and N. Funamori, ibid. 101, 255502 (2008); C. J. Benmore et al., Phys. Rev. B 81, 054105 (2010); T. Sato and N. Funamori, ibid. 82, 184102 (2010); D. Wakabayashi et al., ibid. 84, 144103 (2011).

[18] E. Rapoport, J. Chem. Phys. 46, 2891 (1967); H. Tanaka, Phys. Rev. E 62, 6968 (2000).

[19] S. Rastogi, M. Newman, and A. Keller, Nature (London) 353, 55 (1991); G. W. H. Höhne, S. Rastogi, and B. Wunderlich, Polymer 41, 8869 (2000).

[20] S. Okumura et al., Polymer 37, 2285 (1996).

[21] J. H. Griffith and B. G. Rånby, J. Polym. Sci. 44, 369 (1960).

[22] P. Zoller, J. Appl. Polym. Sci. 21, 3129 (1977).
[23] H. Kusanagi et al., J. Polym. Sci. Pol. Phys. 16, 131 (1978).

[24] A. C. Puleo, D. R. Paul, and P. K. Wong, Polymer 30, 1357 (1989).

[25] M.-H. Kim, J. D. Londono, and A. Habenschuss, J. Polym. Sci. Pol. Phys. 38, 2480 (2000).

[26] Dr. Takehisa Matsukawa (Juntendo University) kindly conducted the atomic absorption spectrometry measurements.

[27] M. A. Belarra et al., J. Anal. At. Spectrom. 1, 141 (1986).

[28] Y. Ohishi et al., High Press. Res. 28, 163 (2008).

[29] H. K. Mao et al., J. Geophys. Res. 96, 8069 (1991).

[30] A. Lacam and C. Chateau, J. Appl. Phys. 66, 366 (1989); F. Datchi et al., High Press. Res. 27, 447 (2007).

[31] P. Zoller et al., Rev. Sci. Instrum. 47, 948 (1976).

[32] The measurements were performed in order of increasing and decreasing pressure at $290{ }^{\circ} \mathrm{C}$, followed by increasing and decreasing pressure at $280{ }^{\circ} \mathrm{C}$. The difference of $I_{1} / I_{2}$ between 280 and $290{ }^{\circ} \mathrm{C}$ [Fig. 4(a)] would be due to a small leakage of the molten sample when decreasing temperature near ambient pressure. The difference could be also due to graphite grains (gasket material) that entered the sample chamber gradually and reached to the sample position for the measurements at $280{ }^{\circ} \mathrm{C}$.

[33] The reversibility indicates that the sample is free from radiation damages. Moreover, the sample crystallized as phase I after the high-temperature experiments. This also suggests that there are no significant radiation damages.

[34] F. Birch, J. Geophys. Res. 83, 1257 (1978).

[35] In this paper, we discuss the similarity of the behavior between $\mathrm{SiO}_{2}$ glass and P4MP1 melt. It may be worth pointing out that detailed studies have clarified that the transition in $\mathrm{SiO}_{2}$ glass is the first order.

[36] The error bars in Fig. 1 show the accuracy of pressure in the diffraction experiments. Although the accuracy is not very high, the precision is sufficiently high and is comparable to the size of the symbols plotted in Fig. 4. See the experimental section for details.

[37] J. C. Phillips, J. Non-Cryst. Solids 43, 37 (1981); D. L. Price et al., J. Phys.: Condens. Matter 1, 1005 (1989).

[38] S. R. Elliott, J. Phys.: Condens. Matter 4, 7661 (1992).

[39] A. C. Wright, Phys. Chem. Glasses 49, 103 (2008).

[40] Q. Mei et al., Phys. Rev. B 78, 144204 (2008).

[41] J. R. Katz, Trans. Faraday Soc. 32, 77 (1936).

[42] G. R. Mitchell and A. H. Windle, Polymer 25, 906 (1984).

[43] R. L. Miller and R. F. Boyer, J. Polym. Sci. Pol. Phys. 22, 2021 (1984).

[44] G. Floudas, K. Mpoukouvalas, and P. Papadopoulos, J. Chem. Phys. 124, 074905 (2006).

[45] A. Arbe et al., Soft Matter 4, 1792 (2008).

[46] S. Krimm and A. V. Tobolsky, J. Polymer Sci. 6, 667 (1951).

[47] S. Beret and J. M. Prausnitz, Macromolecules 8, 536 (1975).

[48] C. S. J. van Hooy-Corstjens, G. W. H. Höhne, and S. Rastogi, Macromolecules 38, 1814 (2005). 